\title{
Communications
}

2015; 3(1): 11-23

Published online July 23, 2015 (http://www.sciencepublishinggroup.com/j/com) doi: 10.11648/j.com.20150301.12

\section{Investigating Space Overhead by IPSec on IPv4 and IPv6 Communication Protocols}

\author{
Muhammed Nura Yusuf, Ali Mohammed Baba \\ Department of Mathematical Sciences, Faculty of Science, Abubakar Tafawa Balewa University Bauchi
}

Email address:

yudasco@yahoo.com (M. N. Yusuf), ambabastas@gmail.com (A. M. Baba)

To cite this article:

Muhammed Nura Yusuf, Ali Mohammed Baba. Investigating Space Overhead by IPSec on IPv4 and IPv6 Communication Protocols. Communications. Vol. 3, No. 1, 2015, pp. 11-23. doi: 10.11648/j.com.20150301.12

\begin{abstract}
IPsec, an internet layer three-security protocol suite is often characterised with introducing an additional space and processing overhead when implemented on a network for secured communication using either IPv4 or IPv6. The use of IPsec on IPv4 is an alternative that offers solutions and addresses the security vulnerabilities in network layer of the OSI and TCP/IP protocol stack. In IPv6, IPsec is one among many other features added to the earlier internet protocol to enhance efficiency and security. Analysis in this research aim at observing the effect of additional space overhead incurred by internet protocols version 4 and 6 (IPv4, IPv6) as a result of selected IPsec configuration in relation to payload size in transport and tunnel mode of IPsec. It was observed that the cost of IPsec added overhead is relatively small when smaller packet sizes are involved for both protocols comparison with large packet sizes that were IPsec protected with the same configuration as the smaller packet, unless in the cases whereby the packet was very large which has to be fragmented. It is therefore, a guide for network administrators to trade up between processing cost and larger address space among other improvements specifically for transmission involving larger IP packets.
\end{abstract}

Keywords: IPv4, IPv6, IPsec, Transport Mode, Tunnel Mode

\section{Introduction}

Analysis of IPSec overheads has generated significant amount of research interest over the years. There are various publications of technical and peer reviewed papers and thesis that already worked on the area. This discussion and analysis cover areas such as basic network protocol performance ranging from protocol latency, throughput, CPU utilization of protocols, to TCP/IP IPSec protocol processing overheads

(Meenakshi 2006) in his work described protocol latency as the duration period whereby an IP Packet or datagram is kept on hold by certain network protocol or predecessor layer for processing prior to pushing it further to awaiting network component or next protocol. It was therefore, narrated that this brings about a processing overheads that the protocol introduces to the performance of the entire network, thus, degrading its efficiency, exerting more effect on a multilayer protocol hierarchy. According to him, this latency can be either start latency or stop latency. Start latency is described as the time between the initial bit of the payload data arriving at the top of the stack and it is part of stop latency. Stop latency refers to the time interval between the initial bit of the data payload arriving at the header of the protocol stack and up to the last bit of the payload going out through the bottom of the stack (Meenakshi 2006).

Hence in mathematical expressions it was given as:

Start latency $\Delta$ tstart $=($ time difference between $\Delta I$ reaches SPn and $\Delta i$ exits SPi)

Stop latency $\Delta$ tstop $=($ time difference between $\Delta$ reaches $S P n$ and $\Delta n$ exits $S P i)$

Total latency $\Delta$ ttot $=(\Delta$ tstop $($ server side $)+\Delta$ tstop $($ client side) + transmission delay)

Another performance parameter is throughput. In the same work, (Meenakshi 2006) described it as the amount of transmitting data a channel can accommodate and process it at a particular point in time; it is measured in Kbps. This is seriously affected as a result of any additional overheads that might be encountered or introduced on a network (Meenakshi 2006).

On the other hand, (Elkeelany et al 2002), on analytical performance investigation of IPSec overheads approached it with respect to time and space complexity. Ciphering 
algorithms such as triple DES and authentication algorithms like HMAC-MD5 and HMAC-SHA1 were investigated in relation to AH and ESP, in the two modes of IPSec. Their overall effects were determined and compared.

(Seiji et al 2000) approached it experimentally and investigated the performance of IPSec over IPV4 with the objective of evaluating the end-to-end throughput of transmitting a very large data and real time traffic such as video application. Here is what he had found summarized in table 1 .

Table 1.0 Summarized Result of End-To-End Throughput of Transmitting a Very Large Data and Real Time Traffic Such As Video Application

\begin{tabular}{|c|c|}
\hline Measurement & Result/Comment \\
\hline Larger Data Transfer & $\begin{array}{l}\text { The throughput deteriorates to } 1 / 9 \\
\text { when authentication and encryption } \\
\text { are enforced. }\end{array}$ \\
\hline Digital Video Transfer & $\begin{array}{l}\text { While here, the throughput } \\
\text { depreciates to } 1 / 10 \text { when } \\
\text { authentication and encryption are } \\
\text { enforced. }\end{array}$ \\
\hline $\begin{array}{l}\text { The overall end-to-end throughput } \\
\text { deterioration discrepancy between } \\
\text { the two IP protocols. }\end{array}$ & The difference is minor. \\
\hline $\begin{array}{l}\text { With IPSec enabled the divergence } \\
\text { with respect to end-to-end } \\
\text { throughput of TCP and UDP } \\
\text { protocols. } \\
\text { However, the throughput reduction } \\
\text { in relation to response mode is } \\
\text { substantially higher in than in the } \\
\text { stream mode }\end{array}$ & It is equals \\
\hline
\end{tabular}

(Seiji et al 2002)

Similarly (George 2003) concentrated on looking at the constituents of the overheads IPv4 with IPSec enabled introduce to an email and web application over a wired and wireless communication channel using HMAC-MD5 and HMAC-SHA1 for authentication and 3DES for encryption. Below is what they had to presents.

In the case of wired Network channel:

1. With the amalgamation of different encryption and authentication algorithms for transmitting HTTP and SMTP application protocol with file size higher than $10 \mathrm{~kb}$, the number of transaction increase by $5 \%$ between client and the server.

2. While in the events of client machine speed slower than the server, the number of time at which transaction takes place are $22 \%$ for SMTP and $10 \%$ for HTTP protocol

3. The network load increased between the range of 20 to $30 \%$ for $1 \mathrm{~KB}$ and about $5 \%$ for $10 \mathrm{MB}$ when HTTP is in Play

4. While for SMTP the network load experience an additional increase of 31 to $40 \%$ for $1 \mathrm{~KB}$ and 6 to 12 $\%$ for $10 \mathrm{MB}$

5. Meanwhile the increase in transfer time is between 18 to $52 \%$ for $1 \mathrm{~KB}$ and 6 to $12 \%$ for $10 \mathrm{MB}$ files

6. While SMTP recoded an increase in transfer time with 3 to $11 \%$ for $1 \mathrm{~KB}$ and 1 to $6 \%$ with $10 \mathrm{MB}$ files
In the other case, the Wireless Network channel:

1. Even with the IPSec in play, there is no increase in the number of transactions

2. Network load is uniform for both slow and fast client

3. But transfer time is increased by about 6 to 285 for $10 \mathrm{~KB}$ and 5 to $6 \%$ for $1 \mathrm{MB}$ with HTTP while 6 to $14 \%$ for $10 \mathrm{~KB}$ and $3 \%$ for $1 \mathrm{MB}$ with SMTP. (George 2003)

On a similar approached (Lin 2003) investigated a VPN throughput of routers setup with IPv4 using IPSec technology, considering various file size to measure the overall throughput for FTP traffic and HTTP application protocols. For authenticating the connection HMAC-MD5 and HMAC-SHA1 algorithms were used while DES and 3DES were used for encryption. The outcome of the work is summarized as follows:

- Throughput disparity between FTP and HTTP protocol for file size of $100 \mathrm{MB}$ indicates that FTP Protocol is greater than HTTP throughput by 1 to $3 \%$

- Using HMAC-MD5 authentication of file size $1 \mathrm{MB}$ the throughput of HTTP exceed the one of FTP by 1 to $2 \%$. While it is almost equal when HMAC-SHA1 is used

- In summary the average deterioration of the throughput is $1 / 3$ with HMAC-MD5, 1/3.5 for HMAC-SHA1,1/7 for DES plus MD5,1/8 for DES plus SHA1, 1/9 for 3DES plus MD5 and lastly $1 / 10$ for 3Des plus SHA1 to all the application (FTP and HTTP)

For real time application such as VoIP and video conferencing, (Klause 2005) looked at the effects overheads have on measurement parameters such as data loss, jitter and delay in relation to quality of service. Briefly to summarize their findings, the IPSec overheads do not attribute any effect on perceptual quality, and the measurement parameters investigated did not experience any substantial disparity for packet greater than 256bytes with IPSec enabled or not enabled. (Klause 2005)

\section{IPSec and Its Components}

IPSec is an IP network mechanism that operates at the network layer of the TCP/IP and OSI protocol stack (Mujinga et al 2006). It is a standard that offers security parameters at the network layer of IP base network for secured end-2-end data transmission. It inspects the process of authentication for the communicating parties, and engages the services of various cryptographic algorithms to provide confidentiality and anti-replay attack to the data. IPSec is a tool that can be used to bring virtual private network (VPN) into being in an IPv4-based network (Wenhong et al 2006). The VPN allows you to create a secured and private communicating channel using unsecured and public network medium such as the Internet. The use of IPSec is optional in IPv4-based network, depending on the level of priority given to security at the network layer by users and their application in a given network. IPv4 in its plain form is noted to be vulnerable, susceptible and defenceless to any possible adversary attack targeted at the network layer of the OSI and TCP/IP protocol stack. I.e. attack such as IP spoofing, ping of death and cache 
poisoning (Cheng 2011) etc. But the emergence of IPSec offers IPv4 the option to alleviate such threats and immunize itself against the possible attacks. This is achieved by adding IPSec headers to the IPv4 packets in VPN connections and provides secure tunnelling protocol connection between the communicating parties with the help of its two integral protocols, authentication header protocol (AH) and encapsulating security payload (ESP). However, unlike in IPv4, IPSec is an integral part of IPv6 protocol (Cheng 2011). It is one of the additional features introduced to the protocol to enhance network security. In IPv6 the implementation of IPSec is necessary and is achieved with the help of two sets of protocols; authentication header $(\mathrm{AH})$ and encapsulating security payload (ESP) both of which are integral part of IPSec protocols that offer the choices of selecting desired security services available. Together they make up the IPSec transform, without them IPSec cannot give us the primary service it was developed for (Lammle 2010).

Similarly, it is important to note, that IPSec is compatible to operate in two different modes, the transport mode and the tunnel mode. AH and ESP can operate in both modes, and each of the mode has its own peculiar characteristics and uses that to distinguish itself from the other. The choice of the mode depends on the network design implementation and the path/route of the transmitted data. In transport mode IPSec provide its security to the end-2-end points of the transmission by applying encryption algorithm to the IP packet payload only. In other words, it encrypts the actual datagram only while leaving the IP header as open plain text. Contrary to the transport mode, in IPSec tunnel mode, the encryption algorithm is applied to the entire IP packet, both the payload and the IP header inclusive, and then encapsulates it in a new IP header, this means that no portion of the IP packet is exempted from IPSec protection during transmission, (Mujinga et al 2006), (Christos et al 2006).

As stated earlier, IPSec employ the service of two protocols to ensure the end-2-end secure channel for communication. These includes; Authentication Header (AH) and Encapsulating Security Payload (ESP) (Mujinga et al 2006). The AH contributes to sustain data origin authentication, connectionless integrity and an optional anti-reply service, but without one for confidentiality. AH render these services by generating a one-way hash function that is identical from the sender and the receiver. If the one-way hash function were to change in any way the packet originality couldn't be establish and authenticated, therefore, the packet would be dropped instantly (Lamme 2010). Similarly, for multicast transmission one-way hash function may be merged with a symmetric signature algorithm, hence performance and space constraints make the utilization of such algorithm impracticable, thus hindering it deployment (IETF/RFC 4305). However, the encapsulating security payload (ESP) in the other hand, being a twin of AH in IPSec transform, steps up and fills the gap left by $\mathrm{AH}$. It is a protocol designed to maintain data confidentiality, data origin authentication, connectionless integrity, anti-reply immunity service and adjusted traffic flow confidentiality. ESP can be deployed exclusively or in conjunction with $\mathrm{AH}$ to form a stronger union of IPSec mechanism for securing the data being transmitted through the channel (Mujinga et al 2006).

The diagrams below demonstrate the IPSec authentication header $(\mathrm{AH})$ transport mode and tunnel mode positioning and size for an IPv4 and IPv6 IP packets (IETF/ RFC 4305)

Fig 1. IPv4 with IPSec (AH) Total Header Size, Tunnel Mode 64 Bytes.

\begin{tabular}{|c|c|c|c|c|c|c|}
\hline \multirow{4}{*}{$\begin{array}{l}\text { Original IPv4 Header } \\
\text { total Size }=20 \text { bytes }\end{array}$} & 0-3 & 4-7 & $8-13$ & $14-15$ & $16-18$ & $19-31$ \\
\hline & Version (4 bit) & $\begin{array}{l}\text { Internet Header } \\
\text { Length (4 bit) }\end{array}$ & $\begin{array}{l}\text { Differentiated Services } \\
\text { Code Point ( } 8 \text { bit) }\end{array}$ & $\begin{array}{l}\text { Explicit Congestion } \\
\text { Notification() }\end{array}$ & \multicolumn{2}{|c|}{ Total Length (16 bit) } \\
\hline & \multicolumn{4}{|c|}{ Identification (16 bit) } & Flags (3 bit) & $\begin{array}{l}\text { Fragment } \\
\text { Offset (13 bit) }\end{array}$ \\
\hline & \multicolumn{2}{|c|}{$\begin{array}{l}\text { Time to Live }(8 \text { bit) } \\
\text { Source IP Address (36bit) } \\
\text { Destination IP Address }(36 \text { bit) } \\
\text { Options (if Header Length }>5 \text { ) }\end{array}$} & \multicolumn{2}{|l|}{ Protocol (8 bit) } & \multicolumn{2}{|c|}{ Header checksum (16bit) } \\
\hline $\mathrm{AH}$ & 44 bytes & & & & & \\
\hline User Data & Data () & & & & & \\
\hline
\end{tabular}

Source: IPv4 (IETF/ RFC 4305)

Fig 2. IPv4 with IPSec (ESP) Total Header Size, Tunnel Mode 62 Bytes.

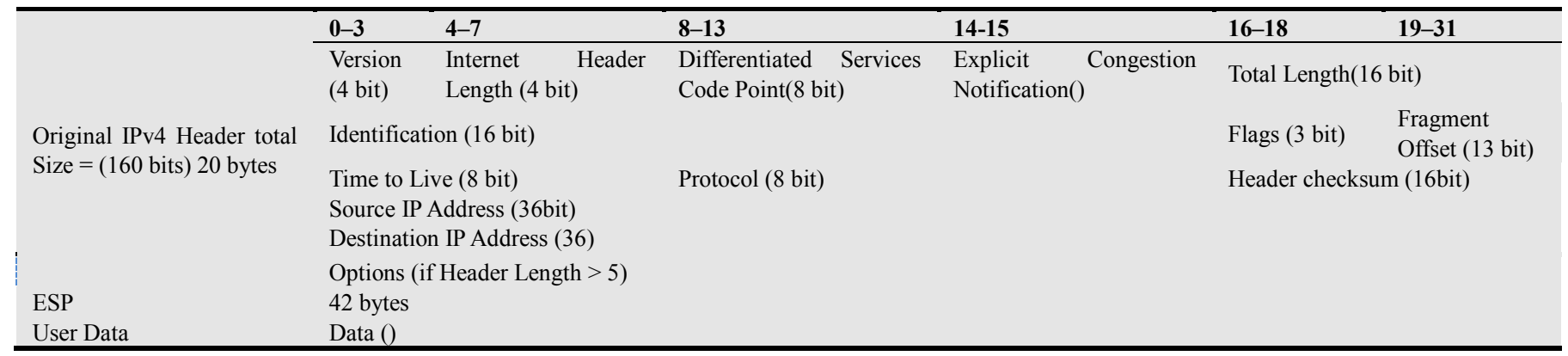

Source: IPv6 (IETF/ RFC 4305) 
Fig 3. IPv6 with IPSec (AH) Total Header Size, Transport Mode 64 Bytes.

\begin{tabular}{lllc}
\hline & Version (4 bits) & Traffic class (8 bits) & Flow label (20 bits) \\
\cline { 2 - 4 } Original & Payload Length (16 bits) & Next Header (8 bits) & Hop Limit (8 bits) \\
IPv6 Header. total size $=320$ bits (40 bytes) & Source address (128 bits) & \\
AH & Destination Address (128 bits) & \\
User Data & 24 byte & \\
\hline
\end{tabular}

Source: IPv6 (IETF/ RFC 4305)

Fig 4. IPv6 with IPSec (ESP) Total Header Size Transport Mode 62 Byte.

\begin{tabular}{llll|}
\hline \multirow{2}{*}{$\begin{array}{l}\text { Original } \\
\text { IPv6 Header. Total size }=320 \text { bits (40 bytes) }\end{array}$} & Version (4 bits) & Traffic class (8 bits) & Flow label (20 bits) \\
\cline { 2 - 4 } & Payload Length (16 bits) & Next Header (8 bits) & Hop Limit (8 bits) \\
ESP & Source address (128 bits) & & \\
User Data & 22 byte & & \\
\hline
\end{tabular}

Source: IPv6 (IETF/ RFC 4305)

Fig 5. IPv6 with IPSec (AH) Total Header Size, Tunnel Mode 84 Bytes.

\begin{tabular}{llll}
\hline & Version (4 bits) & Traffic class (8 bits) & Flow label (20 bits) \\
\cline { 2 - 4 } Original & Payload Length $(16$ bits $)$ & Next Header (8 bits) & Hop Limit (8 bits) \\
IPv6 Header. Total size $=320$ bits $(40$ bytes $)$ & Source address $(128$ bits $)$ & & \\
AH & Destination Address $(128$ bits $)$ & & \\
User Data & 44 byte & & \\
\hline
\end{tabular}

Source: IPv6 (IETF/ RFC 4305)

Fig 6. IPv6 with IPSec (ESP) Total Header Size, Transport Mode 82 Bytes.

\begin{tabular}{llll}
\hline & Version (4 bits) & Traffic class (8 bits) & Flow label (20 bits) \\
\cline { 2 - 4 } Original & Payload Length $(16$ bits $)$ & Next Header $(8$ bits $)$ & Hop Limit $(8$ bits $)$ \\
IPv6 Header. Total size $=320$ bits (40 bytes) & Source address $(128$ bits $)$ & \\
ESP & Destination Address (128 bits) & \\
User Data & 42 byte & \\
\hline
\end{tabular}

Source: IPv6 (IETF/ RFC 4305).

\section{Methodology for the Investigation}

IPSec protected packet increased in final size leads to introduction of space overheads. The space overhead is established due to the IPSec supplementary fields that are further added to the plain IP packets to protect it against network layer attacks. The overheads size is relative to the IPSec security protocols adopted and the mode at which IPSec is setup. The investigation was implemented with different packet size, hence the packet size was determine according to the IPSec configuration parameters used (IPSec transform set) to yield the protected packet size. The protected packet introduced an additional space and processing overheads.

The procedure adopted for investigating the IPSec overheads imposed by IPv4 network and comparing the same experienced in IPv6 -based network communication was investigated in the two different modes of IPSec; Transport mode and Tunnel mode, by numerically quantifying the space overhead using the model developed by Christos et al, 2006 to arrive at the final size of the protected packets. The model is given in table 2 and table 3 .
Nine different IPSec protected user payload files sizes were investigated using different IPSec configuration scenarios in the said modes; (transport mode and tunnel mode respectively). All the files are tested using IPv4 and IPv6. The selection of the files is based on its size. The files are categorized as "small file size", "intermediate file size" and "large file size". The small file sizes are 1byte, 10 byte and 100 byte while the intermediates files are between $1 \mathrm{~kb}$ to $100 \mathrm{~kb}$ and the large files are $1 \mathrm{MB}$ to $100 \mathrm{MB}$.

The results obtained were subjected to R 3.1.3 statistical package for analysis of variance (ANOVA) to determine the contribution of IPSec configuration sets and the effects of the protocols, payload size on the incurred overhead in both the transport and tunnel mode.

The model for the design is given by

$$
\mathrm{AO}=\mu+\mathrm{PTC}+\mathrm{PLS}+\mathrm{IPSec}+\varepsilon
$$

Where,

$A O \rightarrow$ additional overhead

$\mu \rightarrow$ constant independent of PTC, PLS, IPSec

$P T C \rightarrow$ effect of protocol 
$P L S \rightarrow$ effect of payload size

IPsec $\rightarrow$ effect of IPsec configuration set

$\varepsilon \rightarrow$ Error term

Where significant difference among PTC, PLS, and IPsec is observed, means separation technique using Bonferrion method is used to find the different classes in each case.

Table 2.0. Description of the symbols and notations used in the model.

\begin{tabular}{ll}
\hline $\begin{array}{l}\text { Symbol notations used } \\
\text { in the model }\end{array}$ & Description of the symbols and notations \\
\hline AuTESP & $\begin{array}{l}\text { Size of the ATH data field of the ESP protocol } \\
(12 \text { bytes }) \\
\text { Block size of the encryption algorithms } \\
\text { (DES }=8 \text { bytes while AES }=16 \text { bytes })\end{array}$ \\
BL & $\begin{array}{l}\text { ESP }=8 \text { bytes, IPv4 header }=20 \text { bytes, } \\
\text { IPv6 header }=40 \text { bytes and TCP header size }= \\
\text { 20bytes } \\
\text { RESP, HIP, HTCP }\end{array}$ \\
RP (Sd), RL (Sd) & $\begin{array}{l}\text { transport mode and tunnel mode } \\
\text { Actual user packet size in bytes }\end{array}$ \\
Sd & $\begin{array}{l}\text { Total size of IPSec applied packet in transport } \\
\text { and tunnel mode respectively }\end{array}$ \\
SP(Sd), SL(Sd) & ESP Trailer size. \\
\hline
\end{tabular}

(Christos et,al 2006)
Table 3.0. Model to Determine the Size of IPSec Applied User Packet.

\begin{tabular}{ll}
\hline Symbols IPv4 & The formula IPv4 \\
\hline SPESP-CNF(Sd) & Ceil $(\mathrm{Sd}+22) / \mathrm{BL} * \mathrm{BL}+28$ \\
SLESP-CNF(Sd) & Ceil $(\mathrm{Sd}+42) / \mathrm{BL} * \mathrm{BL}+28$ \\
SPESP-ATH (Sd) & Ceil $(\mathrm{Sd}+22) / 4 * 4+40$ \\
SLESP-ATH (Sd) & Ceil $(\mathrm{Sd}+42) / 4 * 4+40$ \\
SP ESP-CNF-ATH (Sd) & Ceil $(\mathrm{Sd}+42) / \mathrm{BL} * \mathrm{BL}+40$ \\
SL ESP-CNF_ATH (Sd) & $\mathrm{Ceil}(\mathrm{Sd}+42) / \mathrm{BL} * \mathrm{BL}+40$ \\
SP AH(Sd) & $\mathrm{Sd}+64$ \\
SLAH (Sd) & $\mathrm{Sd}+84$ \\
IPv6 & $\mathrm{IPv} 6$ \\
SPESP-CNF(Sd) & $\mathrm{Ceil}(\mathrm{Sd}+22) / \mathrm{BL} * \mathrm{BL}+48$ \\
SLESP-CNF(Sd) & $\mathrm{Ceil}(\mathrm{Sd}+62) / \mathrm{BL} * \mathrm{BL}+48$ \\
`SPESP-ATH (Sd) & $\mathrm{Ceil}(\mathrm{Sd}+22) / 4 * 4+60$ \\
SLESP-ATH (Sd) & $\mathrm{Ceil}(\mathrm{Sd}+62) / 4 * 4+60$ \\
SP ESP-CNF-ATH (Sd) & $\mathrm{Ceil}(\mathrm{Sd}+42) / \mathrm{BL} * \mathrm{BL}+60$ \\
SL ESP-CNF_ATH (Sd) & $\mathrm{Ceil}(\mathrm{Sd}+62) / \mathrm{BL} * \mathrm{BL}+60$ \\
SP AH(Sd) & $\mathrm{Sd}+84$ \\
SLAH (Sd) & $\mathrm{Sd}+124$ \\
\hline
\end{tabular}

(Christos et,al 200)

\section{Result of the Investigations: (Summarize in Table)}

Table 4.0. (Small size data): The Overheads Imposed In Transport Mode by 1byte, 10byte and 100byte IPSec Protected User Data.

\begin{tabular}{|c|c|c|c|c|c|c|}
\hline Protocols & $\begin{array}{l}\text { Payload/file } \\
\text { size }\end{array}$ & $\begin{array}{l}\text { Packet size with no IPSec } \\
\text { (bytes) }\end{array}$ & $\begin{array}{l}\text { IPSec configuration } \\
\text { set }\end{array}$ & $\begin{array}{l}\text { IPSec Protected } \\
\text { Packet size (bytes) }\end{array}$ & $\begin{array}{l}\text { Additional } \\
\text { overheads (bytes) }\end{array}$ & $\begin{array}{l}\% \quad \text { Of } \\
\text { overheads }\end{array}$ \\
\hline \multirow{4}{*}{ IPv4 } & \multirow{7}{*}{ 1byte } & \multirow{4}{*}{41} & $\mathrm{AH}$ & 65 & 24 & $58 \%$ \\
\hline & & & ESP-CNF & 60 & 19 & $46 \%$ \\
\hline & & & ESP-ATH & 64 & 23 & $56 \%$ \\
\hline & & & ESP-CNF-ATH & 72 & 31 & $75 \%$ \\
\hline \multirow{3}{*}{ IPv6 } & & \multirow{3}{*}{61} & $\mathrm{AH}$ & 85 & 24 & $39 \%$ \\
\hline & & & ESP-CNF & 80 & 19 & $31 \%$ \\
\hline & & & ESP-CNF-ATH & 92 & 31 & $50 \%$ \\
\hline \multirow{4}{*}{ IPv4 } & \multirow{6}{*}{ 10byte } & \multirow{4}{*}{50} & $\mathrm{AH}$ & 74 & 24 & $48 \%$ \\
\hline & & & ESP-CNF & 60 & 10 & $20 \%$ \\
\hline & & & ESP-ATH & 72 & 22 & $44 \%$ \\
\hline & & & ESP-CNF-ATH & 72 & 27 & $54 \%$ \\
\hline \multirow{3}{*}{ IPV6 } & & \multirow{3}{*}{70} & $\mathrm{AH}$ & 94 & 24 & $34 \%$ \\
\hline & & & ESP-CNF & 80 & 10 & $14 \%$ \\
\hline & & & ESP-CNF-ATH & 92 & 32 & $45 \%$ \\
\hline \multirow{4}{*}{ IPv4 } & & \multirow{4}{*}{140} & $\mathrm{AH}$ & 164 & 24 & $17 \%$ \\
\hline & & & ESP-CNF & 156 & 16 & $11 \%$ \\
\hline & & & ESP-ATH & 164 & 24 & $17 \%$ \\
\hline & & & ESP-CNF-ATH & 168 & 28 & $20 \%$ \\
\hline \multirow{4}{*}{ IPv6 } & 100byte & \multirow{4}{*}{160} & $\mathrm{AH}$ & 184 & 24 & $15 \%$ \\
\hline & & & ESP-CNF & 178 & 16 & $10 \%$ \\
\hline & & & ESP-ATH & 184 & 24 & $15 \%$ \\
\hline & & & ESP-CNF-ATH & 188 & 28 & $17.5 \%$ \\
\hline
\end{tabular}

R Table 4 Result: Analysis of variance (ANOVA) and means separation using Bonferrion method.

$\begin{array}{llllll}\text { Df } & \text { Sum } & \text { Sq } & \text { Mean } & \text { Sq } & \text { F value } \operatorname{Pr}(>\mathrm{F}) \\ \text { PTCSDS } & 2 & 2.0 & 0.98 & 0.154 & 0.8582 \\ \text { PLSDS } & 2 & 39.3 & 19.67 & 3.101 & 0.0727 . \\ \text { IPSSDS } & 3 & 609.8 & 203.28 & 32.051 & 5.35 \mathrm{e}-07 * * * \\ \text { Residuals } & 16 & 101.5 & 6.34 & & \end{array}$




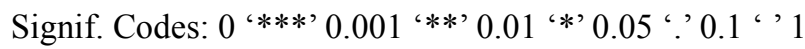

LSD $t$ Test for AOHTSDS

$P$ value adjustment method: bonferroni

Mean Square Error: 6.342438

IPSSDS, means and individual (95\%) CI

$\begin{array}{llllllll} & \text { AOHTSDS } & \text { std } & \mathrm{r} & \text { LCL } & \text { UCL } & \text { Min } & \text { Max } \\ \text { AH } & 24.00000 & 0.000000 & 6 & 21.82044 & 26.17956 & 24 & 24 \\ \text { ESP-ATH } & 23.00000 & 1.000000 & 5 & 20.61241 & 25.38759 & 22 & 24 \\ \text { ESP-CNF } & 16.14286 & 4.810702 & 7 & 14.12498 & 18.16074 & 10 & 23 \\ \text { ESP-CNF-ATH } & 29.50000 & 2.073644 & 6 & 27.32044 & 31.67956 & 27 & 32\end{array}$

alpha: 0.05 ; Df Error: 16

Critical Value of t: 3.008334

Minimum difference changes for each comparison

Means with the same letter are not significantly different.

Groups, Treatments and means

$\begin{array}{lll}\text { a } & \text { ESP-CNF-ATH } & 29.5 \\ \text { b } & \text { AH } & 24 \\ \text { b } & \text { ESP-ATH } & 23 \\ \text { c } & \text { ESP-CNF } & 16.14\end{array}$

Table 5.0. (Small size data): The Overheads Imposed In Tunnel Mode on 1byte, 10byte and 100byte IPSec Protected User Data.

\begin{tabular}{|c|c|c|c|c|c|c|}
\hline Protocols & Payload/file size & $\begin{array}{l}\text { Packet size with no } \\
\text { IPSec (bytes) }\end{array}$ & IPSec configuration set & $\begin{array}{l}\text { IPSec Protected } \\
\text { Packet size (bytes) }\end{array}$ & $\begin{array}{l}\text { Additional } \\
\text { overheads (bytes) }\end{array}$ & $\%$ Of overheads \\
\hline \multirow{4}{*}{ IPv4 } & \multirow{7}{*}{ 1byte } & \multirow{4}{*}{41} & $\mathrm{AH}$ & 85 & 44 & $107 \%$ \\
\hline & & & ESP-CNF & 78 & 35 & $85 \%$ \\
\hline & & & ESP-ATH & 84 & 43 & $104 \%$ \\
\hline & & & ESP-CNF-ATH & 88 & 47 & $114 \%$ \\
\hline \multirow{3}{*}{ IPv6 } & & \multirow{3}{*}{61} & $\mathrm{AH}$ & 124 & 64 & $104 \%$ \\
\hline & & & ESP-CNF & 112 & 51 & $83 \%$ \\
\hline & & & ESP-CNF-ATH & 124 & 63 & $103 \%$ \\
\hline \multirow{4}{*}{ IPv4 } & \multirow{7}{*}{ 10byte } & \multirow{4}{*}{50} & $\mathrm{AH}$ & 94 & 44 & $88 \%$ \\
\hline & & & ESP-CNF & 92 & 42 & $84 \%$ \\
\hline & & & ESP-ATH & 92 & 42 & $84 \%$ \\
\hline & & & ESP-CNF-ATH & 104 & 54 & $108 \%$ \\
\hline \multirow{3}{*}{ IPV6 } & & \multirow{3}{*}{70} & $\mathrm{AH}$ & 134 & 64 & $91 \%$ \\
\hline & & & ESP-CNF & 128 & 58 & $82 \%$ \\
\hline & & & ESP-ATH & 132 & 62 & $88 \%$ \\
\hline \multirow{4}{*}{ IPv4 } & \multirow{8}{*}{ 100byte } & \multirow{4}{*}{140} & $\mathrm{AH}$ & 184 & 44 & $31 \%$ \\
\hline & & & ESP-CNF & 172 & 32 & $22 \%$ \\
\hline & & & ESP-ATH & 184 & 44 & $31 \%$ \\
\hline & & & ESP-CNF-ATH & 184 & 44 & $31 \%$ \\
\hline \multirow{4}{*}{ IPv6 } & & \multirow{4}{*}{160} & $\mathrm{AH}$ & 224 & 64 & $40 \%$ \\
\hline & & & ESP-CNF & 224 & 64 & $40 \%$ \\
\hline & & & ESP-ATH & 224 & 64 & $40 \%$ \\
\hline & & & ESP-CNF-ATH & 236 & 70 & $43 \%$ \\
\hline
\end{tabular}

R Table 5 Result: Analysis of variance (ANOVA) and means separation using Bonferrion method.

$\begin{array}{llllll}\text { Df } & \text { Sum } & \text { Sq } & \text { Mean } & \text { Sq } & \text { F value } \operatorname{Pr}(>\mathrm{F}) \\ \text { PTCTN } & 2 & 2441.1 & 1220.5 & 106.543 & 5.66 \mathrm{e}-10 * * * \\ \text { PLTN } & 2 & 42.5 & 21.2 & 1.855 & 0.188632 \\ \text { IPSTN } & 3 & 375.1 & 125.0 & 10.915 & 0.000378 * * * \\ \text { Residuals } & 16 & 183.3 & 11.5 & & \end{array}$

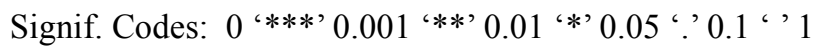

LSD t Test for AOHTN

$P$ value adjustment method: bonferroni

Mean Square Error: 11.45588

PTCTN, means and individual (95\%) CI 


$\begin{array}{llllllll} & \text { AOHTN } & \text { std } & \text { r } & \text { LCL } & \text { UCL } & \text { Min } & \text { Max } \\ \text { IPv4 } & 42.91667 & 5.468228 & 12 & 40.84538 & 44.98795 & 32 & 54 \\ \text { IPv6 } & 63.00000 & 5.215362 & 11 & 60.83661 & 65.16339 & 51 & 70 \\ \text { IPV6 } & 64.00000 & \text { NA } & 1 & 56.82485 & 71.17515 & 64 & 64\end{array}$

alpha: 0.05 ; Df Error: 16

Critical Value of t: 2.673032

Minimum difference changes for each comparison

Means with the same letter are not significantly different.

Groups, Treatments and means

a

a

b

LSD t Test for AOHTN

$P$ value adjustment method: bonferroni

Mean Square Error: 11.45588

IPSTN, means and individual (95\%) CI

$\begin{array}{lllll} & \text { AOHTN } & \text { std } & \mathrm{r} & \text { LCL } \\ \text { AH } & 54 & 10.95445 & 6 & 51.07076 \\ \text { ESP-ATH } & 53 & 10.99091 & 6 & 50.07076 \\ \text { ESP-CNF } & 47 & 12.80625 & 6 & 44.07076 \\ \text { ESP-CNF- } & 58 & 11.36662 & 6 & 55.07076 \\ \text { ATH } & & & & \end{array}$

alpha: 0.05 ; Df Error: 16

Critical Value of $t: 3.008334$

Least Significant Difference 5.878678

Means with the same letter are not significantly different.

Groups, Treatments and means

$\begin{array}{lll}\text { a } & \text { ESP-CNF-ATH } & 58 \\ \text { a } & \text { AH } & 54 \\ \text { a } & \text { ESP-ATH } & 53 \\ \text { b } & \text { ESP-CNF } & 47\end{array}$

64

63

42.92

Table 6.0. The Overheads Imposed In Transport Modes by $1 \mathrm{~kb}, 10 \mathrm{~kb}$ and $100 \mathrm{~kb}$ IPSec Protected User Data.

\begin{tabular}{|c|c|c|c|c|c|c|c|}
\hline Protocols & Payload/file size & $\begin{array}{l}\text { Packet size with } \\
\text { no IPSec (bytes) }\end{array}$ & $\begin{array}{l}\text { IPSec configuration } \\
\text { set }\end{array}$ & $\begin{array}{l}\text { IPSec Protected } \\
\text { Packet size (bytes) }\end{array}$ & $\begin{array}{l}\text { Additional } \\
\text { overheads } \\
\text { (bytes) }\end{array}$ & $\begin{array}{l}\% \\
\text { overheads }\end{array}$ & Of \\
\hline \multirow{5}{*}{ IPv4 } & \multirow{8}{*}{$1 \mathrm{~kb}$ (1024 byte) } & \multirow{5}{*}{1064} & $\mathrm{AH}$ & 1088 & 24 & $2.3 \%$ & \\
\hline & & & ESP-CNF & 1084 & 20 & $1.9 \%$ & \\
\hline & & & ESP-CNF & 1088 & 24 & $2.3 \%$ & \\
\hline & & & ESP-CNF-ATH & 1096 & 32 & $3.0 \%$ & \\
\hline & & & $\mathrm{AH}$ & 1108 & 24 & $2.2 \%$ & \\
\hline \multirow{3}{*}{ IPv6 } & & \multirow{3}{*}{1084} & ESP-CNF & 1104 & 20 & $1.8 \%$ & \\
\hline & & & ESP-ATH & 1108 & 24 & $2.2 \%$ & \\
\hline & & & ESP-CNF-ATH & 1116 & 32 & $3.0 \%$ & \\
\hline \multirow{4}{*}{ IPv4 } & \multirow{8}{*}{ 10kb (10240 byte) } & \multirow{4}{*}{10277} & $\mathrm{AH}$ & 10301 & 24 & $0.23 \%$ & \\
\hline & & & ESP-CNF & 10300 & 23 & $0.22 \%$ & \\
\hline & & & ESP-ATH & 10300 & 23 & $0.22 \%$ & \\
\hline & & & ESP-CNF-ATH & 10312 & 35 & $0.34 \%$ & \\
\hline \multirow{4}{*}{ IPV6 } & & \multirow{4}{*}{10297} & $\mathrm{AH}$ & 10321 & 24 & $0.23 \%$ & \\
\hline & & & ESP-CNF & 10320 & 23 & $0.22 \%$ & \\
\hline & & & ESP-ATH & 10322 & 23 & $0.22 \%$ & \\
\hline & & & ESP-CNF-ATH & 10322 & 35 & $0.23 \%$ & \\
\hline \multirow{4}{*}{ IPv4 } & \multirow{8}{*}{$100 \mathrm{~kb}$ (102400 byte) } & \multirow{4}{*}{102440} & $\mathrm{AH}$ & 102504 & 64 & $0.06 \%$ & \\
\hline & & & ESP-CNF & 102460 & 20 & $0.02 \%$ & \\
\hline & & & ESP-ATH & 102464 & 24 & $0.02 \%$ & \\
\hline & & & ESP-CNF-ATH & 102472 & 32 & $0.03 \%$ & \\
\hline \multirow{4}{*}{ IPv6 } & & \multirow{4}{*}{102460} & $\mathrm{AH}$ & 102524 & 64 & $0.06 \%$ & \\
\hline & & & ESP-CNF & 102524 & 20 & $0.02 \%$ & \\
\hline & & & ESP-ATH & 102484 & 24 & $0.02 \%$ & \\
\hline & & & ESP-CNF-ATH & 102492 & 32 & $0.03 \%$ & \\
\hline
\end{tabular}

R Table 6 Result: Analysis of variance (ANOVA) and means separation using Bonferrion method. 


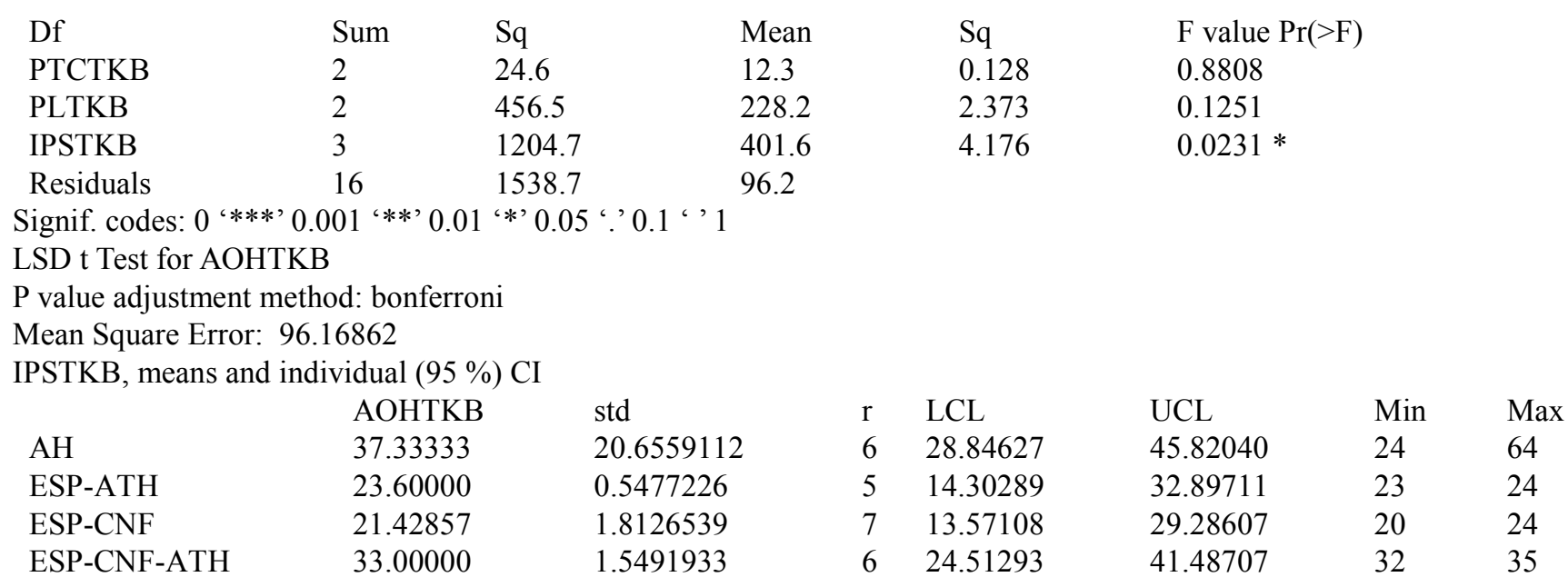

alpha: 0.05 ; Df Error: 16

Critical Value of t: 3.008334

Minimum difference changes for each comparison

Means with the same letter are not significantly different.

Groups, Treatments and means

$\begin{array}{lll}\text { a } & \text { AH } & 37.33 \\ \text { a } & \text { ESP-CNF-ATH } & 33 \\ \text { a } & \text { ESP-ATH } & 23.6 \\ \text { a } & \text { ESP-CNF } & 21.43\end{array}$

Table 7.0. The Overheads Imposed In Tunnel Mode by $1 \mathrm{~kb}, 10 \mathrm{~kb}$ and $100 \mathrm{~kb}$ IPSec Protected User Data.

\begin{tabular}{|c|c|c|c|c|c|c|c|}
\hline Protocols & Payload/file size & $\begin{array}{l}\text { Packet size with no } \\
\text { IPSec (bytes) }\end{array}$ & IPSec configuration set & $\begin{array}{l}\text { IPSec Protected } \\
\text { Packet size (bytes) }\end{array}$ & $\begin{array}{l}\text { Additional } \\
\text { overheads (bytes) }\end{array}$ & $\begin{array}{l}\% \\
\text { overheads }\end{array}$ & Of \\
\hline \multirow{4}{*}{ IPv4 } & \multirow{8}{*}{$1 \mathrm{~kb}$ (1024 byte) } & \multirow{4}{*}{1064} & $\mathrm{AH}$ & 1108 & 44 & $4.14 \%$ & \\
\hline & & & ESP-CNF & 1100 & 36 & $3.38 \%$ & \\
\hline & & & ESP-ATH & 1108 & 44 & $4.14 \%$ & \\
\hline & & & ESP-CNF-ATH & 1112 & 48 & $4.51 \%$ & \\
\hline \multirow{4}{*}{ IPv6 } & & \multirow{4}{*}{1084} & $\mathrm{AH}$ & 1148 & 64 & $5.90 \%$ & \\
\hline & & & ESP-CNF & 1136 & 52 & $4.80 \%$ & \\
\hline & & & ESP-ATH & 1148 & 64 & $5.90 \%$ & \\
\hline & & & ESP-CNF-ATH & 1132 & 48 & $4.43 \%$ & \\
\hline \multirow{4}{*}{ IPv4 } & \multirow{8}{*}{ 10kb (10240 byte) } & \multirow{4}{*}{10277} & $\mathrm{AH}$ & 10321 & 44 & $0.43 \%$ & \\
\hline & & & ESP-CNF & 10316 & 39 & $0.38 \%$ & \\
\hline & & & ESP-ATH & 10320 & 43 & $0.42 \%$ & \\
\hline & & & ESP-CNF-ATH & 10328 & 51 & $0.49 \%$ & \\
\hline \multirow{4}{*}{ IPV6 } & & \multirow{4}{*}{10297} & $\mathrm{AH}$ & 10361 & 64 & $0.62 \%$ & \\
\hline & & & ESP-CNF & 10352 & 55 & $0.53 \%$ & \\
\hline & & & ESP-ATH & 10360 & 63 & $0.61 \%$ & \\
\hline & & & ESP-CNF-ATH & 10364 & 67 & $0.65 \%$ & \\
\hline \multirow{4}{*}{ IPv4 } & \multirow{8}{*}{$100 \mathrm{~kb}$ (102400 byte) } & \multirow{4}{*}{102440} & $\mathrm{AH}$ & 102484 & 44 & $0.04 \%$ & \\
\hline & & & ESP-CNF & 102476 & 36 & $0.03 \%$ & \\
\hline & & & ESP-ATH & 102484 & 44 & $0.04 \%$ & \\
\hline & & & ESP-CNF-ATH & 102524 & 48 & $0.05 \%$ & \\
\hline \multirow{4}{*}{ IPv6 } & & \multirow{4}{*}{102460} & $\mathrm{AH}$ & 102524 & 64 & $0.06 \%$ & \\
\hline & & & ESP-CNF & 102512 & 52 & $0.05 \%$ & \\
\hline & & & ESP-ATH & 102524 & 64 & $0.06 \%$ & \\
\hline & & & ESP-CNF-ATH & 102524 & 64 & $0.06 \%$ & \\
\hline
\end{tabular}

R Table 7 Result: Analysis of variance (ANOVA) and means separation using Bonferrion method.

$\begin{array}{llllll}\text { Df } & \text { Sum } & \text { Sq } & \text { Mean } & \text { Sq } & \text { F value } \operatorname{Pr}(>\mathrm{F}) \\ \text { PTCTNKB } & 3 & 640.5 & 213.50 & 2.471 & 0.0992 \\ \text { PLTNKB } & 2 & 0.0 & 0.00 & 0.000 & 1.0000 \\ \text { IPSTNKB } & 2 & 325.3 & 162.67 & 1.882 & 0.1844 \\ \text { Residuals } & 16 & 1382.7 & 86.42 & & \end{array}$

Signif. codes: $0^{‘ * * *} 0.001^{\prime * *}, 0.01^{\prime *} *$ 
Table 8.0. (Large size data): The Overheads Imposed in Transport Modes by 1Mb, 10Mb and 100Mb IPSec Protected User Data.

\begin{tabular}{|c|c|c|c|c|c|c|c|}
\hline Protocol & $\begin{array}{l}\text { Payload/file size } \\
\text { in byte }\end{array}$ & $\begin{array}{l}\text { Packet size with no } \\
\text { IPSec in bytes }\end{array}$ & IPSec configuration set & $\begin{array}{l}\text { IPSec Protected } \\
\text { Packet size in bytes }\end{array}$ & $\begin{array}{l}\text { Additional } \\
\text { overhead in bytes }\end{array}$ & $\begin{array}{l}\text { \% } \\
\text { overheads } \\
\end{array}$ & Of \\
\hline \multirow{4}{*}{ IPv4 } & \multirow{8}{*}{$\begin{array}{l}(1 \mathrm{MB}) \\
1048576 \text { bytes }\end{array}$} & \multirow{4}{*}{1048616} & $\mathrm{AH}$ & 1048640 & 24 & $0.002 \%$ & \\
\hline & & & ESP-CNF & 1048336 & 20 & $0.001 \%$ & \\
\hline & & & ESP-CNF & 1048640 & 24 & $0.002 \%$ & \\
\hline & & & ESP-CNF-ATH & 1048648 & 32 & $0.003 \%$ & \\
\hline \multirow{4}{*}{ IPv6 } & & \multirow{4}{*}{1048636} & $\mathrm{AH}$ & 1048660 & 24 & $0.002 \%$ & \\
\hline & & & ESP-CNF & 1048656 & 20 & $0.001 \%$ & \\
\hline & & & ESP-ATH & 1048660 & 24 & $0.002 \%$ & \\
\hline & & & ESP-CNF-ATH & 1048668 & 32 & $0.003 \%$ & \\
\hline \multirow{4}{*}{ IPv4 } & \multirow{8}{*}{$\begin{array}{l}(10 \mathrm{MB}) \\
10485760 \text { byte }\end{array}$} & \multirow{4}{*}{10485800} & $\mathrm{AH}$ & 10485824 & 24 & $0.0002 \%$ & \\
\hline & & & ESP-CNF & 10485820 & 20 & $0.0001 \%$ & \\
\hline & & & ESP-ATH & 10485824 & 24 & $0.0002 \%$ & \\
\hline & & & ESP-CNF-ATH & 10485832 & 32 & $0.0003 \%$ & \\
\hline \multirow{4}{*}{ IPV6 } & & \multirow{4}{*}{10485820} & $\mathrm{AH}$ & 10485845 & 25 & $0.0002 \%$ & \\
\hline & & & ESP-CNF & 10485840 & 20 & $0.0001 \%$ & \\
\hline & & & ESP-ATH & 10485844 & 24 & $0.0002 \%$ & \\
\hline & & & ESP-CNF-ATH & 10485844 & 32 & $0.0003 \%$ & \\
\hline \multirow{4}{*}{ IPv4 } & & \multirow{4}{*}{104857640} & $\mathrm{AH}$ & 104857685 & 24 & $0.00002 \%$ & \\
\hline & & & ESP-CNF & 104857660 & 20 & $0.00001 \%$ & \\
\hline & & & ESP-ATH & 104857664 & 24 & $0.00001 \%$ & \\
\hline & $(100 \mathrm{MB})$ & & ESP-CNF-ATH & 104857672 & 32 & $0.00003 \%$ & \\
\hline \multirow{4}{*}{ IPv6 } & 10485760byte & \multirow{4}{*}{104857660} & $\mathrm{AH}$ & 104857684 & 24 & $0.00002 \%$ & \\
\hline & & & ESP-CNF & 104857680 & 20 & $0.00001 \%$ & \\
\hline & & & ESP-ATH & 104857684 & 24 & $0.00002 \%$ & \\
\hline & & & ESP-CNF-ATH & 104857692 & 32 & $0.00003 \%$ & \\
\hline
\end{tabular}

R Table 8 Result: Analysis of variance (ANOVA) and means separation using Bonferrion method.

$\begin{array}{llllll}\text { Df } & \text { Sum } & \text { Sq } & \text { Mean } & \text { Sq } & \text { F value } \operatorname{Pr}(>\mathrm{F}) \\ \text { PTCSD } & 2 & 0.0 & 0.02 & 0.033 & 0.968 \\ \text { PLCSD } & 2 & 0.1 & 0.05 & 0.072 & 0.930 \\ \text { IPSSD } & 3 & 442.7 & 147.58 & 195.913 & 8.03 \mathrm{e}-13 \text { *** } \\ \text { Residuals } & 16 & 12.1 & 0.75 & & \end{array}$

$--$

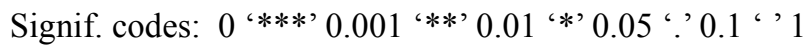

LSD t Test for AOHSD

$P$ value adjustment method: bonferroni

Mean Square Error: 0.753304

IPSSD, means and individual (95\%) CI

$\begin{array}{llllllll} & \text { AOHSD } & \text { std } & \text { r } & \text { LCL } & \text { UCL } & \text { Min } & \text { Max } \\ \text { AH } & 24.16667 & 0.4082483 & 6 & 23.41552 & 24.91782 & 24 & 25 \\ \text { ESP-ATH } & 24.00000 & 0.0000000 & 5 & 23.17716 & 24.82284 & 24 & 24 \\ \text { ESP-CNF } & 20.57143 & 1.5118579 & 7 & 19.87600 & 21.26686 & 20 & 24 \\ \text { ESP-CNF-ATH } & 32.00000 & 0.0000000 & 6 & 31.24885 & 32.75115 & 32 & 32\end{array}$

alpha: 0.05 ; Df Error: 16

Critical Value of t: 3.008334

Minimum difference changes for each comparison

Means with the same letter are not significantly different.

Groups, Treatments and means

$$
\begin{aligned}
& \mathrm{a} \\
& \mathrm{b} \\
& \mathrm{b}
\end{aligned}
$$$$
\text { ESP-CNF-ATH }
$$ 
Table 9.0. (Large size data): The Overheads Imposed in Tunnel Modes by 1Mb, 10Mb and 100Mb IPSec Protected User Data.

\begin{tabular}{|c|c|c|c|c|c|c|}
\hline Protocols & Payload/file size (byte) & $\begin{array}{l}\text { Packet size with } \\
\text { no IPSec (bytes) }\end{array}$ & $\begin{array}{l}\begin{array}{l}\text { IPSec configuration } \\
\text { set }\end{array} \\
\end{array}$ & $\begin{array}{ll}\text { IPSec } & \text { Protected } \\
\text { Packet size (bytes) }\end{array}$ & $\begin{array}{l}\text { Additional } \\
\text { overheads (bytes) }\end{array}$ & $\begin{array}{l}\% \\
\text { overheads }\end{array}$ \\
\hline \multirow{4}{*}{ IPv4 } & & \multirow{4}{*}{1048616} & $\mathrm{AH}$ & 1048660 & 44 & $0.004 \%$ \\
\hline & & & ESP-CNF & 1048648 & 32 & $0.003 \%$ \\
\hline & & & ESP-CNF & 1048660 & 44 & $0.004 \%$ \\
\hline & $(1 \mathrm{MB})$ & & ESP-CNF-ATH & 1048664 & 48 & $0.004 \%$ \\
\hline \multirow{4}{*}{ IPv6 } & 1048576bytes & \multirow{4}{*}{1048636} & $\mathrm{AH}$ & 1048704 & 68 & $0.006 \%$ \\
\hline & & & ESP-CNF & 1048688 & 52 & $0.005 \%$ \\
\hline & & & ESP-ATH & 1048700 & 64 & $0.006 \%$ \\
\hline & & & ESP-CNF-ATH & 1048700 & 64 & $0.006 \%$ \\
\hline \multirow{4}{*}{ IPv4 } & & \multirow{4}{*}{10485800} & $\mathrm{AH}$ & 10485844 & 44 & $0.0004 \%$ \\
\hline & & & ESP-CNF & 10485836 & 36 & $0.0003 \%$ \\
\hline & & & ESP-ATH & 10485844 & 44 & $0.0004 \%$ \\
\hline & $(10 \mathrm{MB})$ & & ESP-CNF-ATH & 10485848 & 48 & $0.0004 \%$ \\
\hline \multirow{4}{*}{ IPV6 } & 10485760 bytes & \multirow{4}{*}{10485820} & $\mathrm{AH}$ & 10485888 & 68 & $0.0006 \%$ \\
\hline & & & ESP-CNF & 10485872 & 52 & $0.0005 \%$ \\
\hline & & & ESP-ATH & 10485884 & 64 & $0.0006 \%$ \\
\hline & & & ESP-CNF-ATH & 10485884 & 64 & $0.0006 \%$ \\
\hline \multirow{4}{*}{ IPv4 } & & \multirow{4}{*}{104857640} & $\mathrm{AH}$ & 104857684 & 44 & $0.00004 \%$ \\
\hline & & & ESP-CNF & 104857676 & 36 & $0.00003 \%$ \\
\hline & & & ESP-ATH & 104857684 & 44 & $0.00004 \%$ \\
\hline & $(100 \mathrm{MB})$ & & ESP-CNF-ATH & 104857688 & 48 & $0.00004 \%$ \\
\hline \multirow{4}{*}{ IPv6 } & 10485760 bytes & \multirow{4}{*}{104857660} & $\mathrm{AH}$ & 104857728 & 68 & $0.00006 \%$ \\
\hline & & & ESP-CNF & 104857712 & 52 & $0.00005 \%$ \\
\hline & & & ESP-ATH & 104857724 & 64 & $0.00006 \%$ \\
\hline & & & ESP-CNF-ATH & 104857724 & 64 & $0.00006 \%$ \\
\hline
\end{tabular}

R Table 9 Result: Analysis of variance (ANOVA) and means separation using Bonferrion method.

$\begin{array}{llllll}\text { Df } & \text { Sum } & \text { Sq } & \text { Mean } & \text { Sq } & \text { F value } \operatorname{Pr}(>\mathrm{F}) \\ \text { ptct } & 3 & 2255.0 & 751.7 & 77.593 & 2.31 \mathrm{e}-09 * * * \\ \text { pls } & 2 & 27.7 & 13.8 & 1.428 & 0.271 \\ \text { ipst } & 3 & 545.3 & 181.8 & 18.765 & 2.45 \mathrm{e}-05 * * * \\ \text { Residuals } & 15 & 145.3 & 9.7 & & \end{array}$

$--$

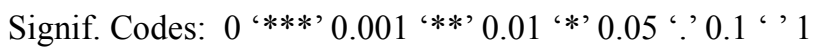

LSD t Test for aoht

$P$ value adjustment method: bonferroni

Mean Square Error: 9.68732

ptct, means and individual ( $95 \%) \mathrm{CI}$

$\begin{array}{llllllll} & \text { aoht } & \text { std } & \mathrm{r} & \text { LCL } & \text { UCL } & \text { Min } & \text { Max } \\ \text { IPv4 } & 42.66667 & 5.465040 & 6 & 39.95834 & 45.37499 & 32 & 48 \\ \text { IPV4 } & 42.66667 & 5.465040 & 6 & 39.95834 & 45.37499 & 36 & 48 \\ \text { IPv6 } & 63.20000 & 6.572671 & 5 & 60.23318 & 66.16682 & 52 & 68 \\ \text { IPV6 } & 61.14286 & 6.414270 & 7 & 58.63543 & 63.65028 & 52 & 68\end{array}$

alpha: 0.05 ; Df Error: 15

Critical Value of t: 3.036283

Minimum difference changes for each comparison

Means with the same letter are not significantly different.

Groups, Treatments and means

$\begin{array}{lll}\text { a } & \text { IPv6 } & 63.2 \\ \text { a } & \text { IPV6 } & 61.14 \\ \text { b } & \text { IPv4 } & 42.67 \\ \text { b } & \text { IPV4 } & 42.67\end{array}$

LSD t Test for aoht

$P$ value adjustment method: bonferroni

Mean Square Error: 9.68732

ipst, means and individual ( $95 \%) \mathrm{CI}$

$\begin{array}{llllllll} & \text { aoht } & \text { std } & \text { r } & \text { LCL } & \text { UCL } & \text { Min } & \text { Max } \\ \text { AH } & 56.00000 & 13.145341 & 6 & 53.29167 & 58.70833 & 44 & 68\end{array}$




$\begin{array}{lll}\text { ESP-ATH } & 56.00000 & 10.95445 \\ \text { ESP-CNF } & 43.42857 & 8.772251 \\ \text { ESP-CNF-ATH } & 56.00000 & 8.763561 \\ \text { alpha: } 0.05 \text {; Df Error: } 15 & \\ \text { Critical Value of t: } 3.036283 & \\ \text { Minimum difference changes for each comparison } \\ \text { Means with the same letter are not significantly differ } \\ \text { Groups, Treatments and means } \\ \text { a } & \text { AH } \\ \text { a } & \text { ESP-ATH } \\ \text { a } & \text { ESP-CNF- } \\ \text { b } & \text { ESP-CNF } \\ & \\ \text { 5. Observations: Interpretation and } \\ \text { Analysis of Result }\end{array}$

Analysis in this research aim at observing the effect of additional space overhead incurred by internet protocols version 4 and 6 (IPv4, IPv6) as a result of selected IPsec configuration in relation to payload size in transport and tunnel mode of IPsec.

Table 4 shows the analysis of variance on the overhead imposed in transport mode by the said protocols (IPv4 and IPv6) configured with AH, ESP-CNF, ESP- ATH, ESP-CNF and $\mathrm{CNF}-\mathrm{ATH}$ transmitting/transporting a payload size of 1 byte, 10 byte, and 100 byte.

The $\mathrm{R}$ result reveals that the choice of IPsec security configuration is significant at $0.1 \%$ level, but the selection of a particular protocol i.e. IPv4 or IPv6 or payload size is not significant.

However, when the IPsec security configuration are subjected to means separation using Bonferrion method, the result reveals that ESP-CNF has the least added overhead flowed by AH and ESP-ATH having the same performance and finally ESP-CNF-ATH having the highest. Similarly, when the selected IPsec security configurations are compared between IPv4 vs. IPv6 and the result tabulated in table 4, it indicated that from all the scenarios IPv4 introduced higher space overheads than IPv6. Hence, this is only when the IPSec transform set are applied on small user data (payload) that are not fragmented; because the actual IPSec headers size is equal on both protocols but if the files need to be fragmented as in the case of larger files, IPv6 incurred higher overhead. This can be reference to the manner at which IPSec header is implemented on the packets that require fragmentation. In IPv4 the IPSec header is applied only to the initial fragment, while in IPv6 the IPSec header is applied to all the fragmented portions of the packet. Therefore, the total cumulative result suppose IPv6 introduce higher overhead when large data are involved.

Table 5 shows the ANOVA of overhead imposed in tunnel mode by the same variables as in above. The result reveals that the protocols IPv4, IPv6 and IPsec security configuration set are both significant at $0.1 \%$ level while the payload size is not.

$\begin{array}{llll}53.03318 & 58.96682 & 44 & 64 \\ 40.92115 & 45.93600 & 32 & 52 \\ 53.29167 & 58.70833 & 48 & 64\end{array}$

64

64
56
56
56
43.43

Further test of means separation using Bonferrion method for the protocols shows IPv4 has lower added overhead as compared to IPv6

Table 5 also shows the means separation using Bonferrion method of the IPsec security configuration set, with ESPCNF having the lowest added overhead followed by ESPATH. AH and ESP-CNF-ATH incurred the same performance.

However, when the selected file size of 1 byte, 10 byte and 100 byte are factored in, an increase in the overheads is introduced. As can be seen in table 5, on one byte of data the least additional overhead incurred is $85 \%$ when ESP-CNF is used but AH, ESP-CNF and ESP-ATH introduce more than $100 \%$ additional space overhead when IPv4 is used. The situation is similar even with IPv6. The added overhead experienced higher increase compared to the one recorded in transport mode, this increase can be attributed to the fact that in tunnel mode the header and the payload are both encrypted and encapsulated in a newly created packet. Another observation made was that as the payload size increases the overhead begins to reduce slowly, as in the case of 100 byte. With 100 byte IPSec protected data in transport mode, the overhead caused by AH, ESP-CNF and ESP-ATH is $17 \%$ for all in IPv4 and $15 \%, 10 \%, 15 \%$ with IPv6 respectively. On the other hand ESP-CNF-ATH gives $20 \%$ and $17.5 \%$ with IPv4 and IPv6 respectively. The same falls is noticed in tunnel mode.

When the payload sizes are increased to 1 kilobyte, 10 kilobyte and 100 kilobyte, Table 6 shows the ANOVA for the overhead incurred in transport mode by IPsec. The result reveals that only IPsec is significant, this time at 5\% level with ESP-CNF having the least addition compared to the rest when subjected to means separation using Bonferrion method. Table 6 also reveals that with 1 kilobyte, $\mathrm{AH}$ and ESP-CNF show 2.3\% overhead in IPv4 and $0.23 \%, 0.22 \%$ with $10 \mathrm{~kb}$. With $100 \mathrm{~kb}$, only $0.06 \%, 0.02 \%$ and $0.02 \%$ are caused by AH, ESP-CNF and ESP-ATH. Meanwhile with IPV6 in play the same trend is observed as only $2.2 \%$ and $3.0 \%$ overheads are recorded on $1 \mathrm{~kb}$ because of AH, ESPCNF, ESP-ATH and ESP-CNF-ATH respectively. Similarly, with $10 \mathrm{~kb}$ big falls in the overhead is noticed because $\mathrm{AH}$ and ESP-ATH-CNF gives $0.23 \%$, ESP-CNF and ESP-ATH is $0.22 \%$. Bigger reduction is observed when $100 \mathrm{~KB}$ is used. 
The result shows that ESP-CNF and ESP-ATH recorded 0.02 $\%$ overhead while AH and ESP-CNF-ATH recorded $0.06 \%$ and $0.03 \%$ respectively and as usual the overhead incurred in tunnel mode when the same file size is used is slightly greater due to the encapsulation. The means separation using Bonferrion method suggests that this is not a significant difference.

When tunnel mode is considered for the same configuration, Table 7 shows the ANOVA for the overhead imposed; the result reveals that all the three variables i.e. the IP protocols, IPsec configuration set and the payload size are not significant at $5 \%$. Therefore, no further means separation test is required.

Lastly, when very large files are used the space overhead reduced drastically. Table 9 shows the ANOVA for the overhead imposed in transport while transmitting packets of sizes $1 \mathrm{mb}, 10 \mathrm{mb}$ and $100 \mathrm{mb}$. The result reveals that only IPsec is significant at $0.1 \%$.

The means separation using Bonferrion method for the IPsec reveals that ESP-CNF has the least overhead followed by ESP-ATH, AH and lastly ESP-CNF-ATH. This was the observation made when $1 \mathrm{mb}, 10 \mathrm{mb}$ and $100 \mathrm{mb}$ are used as the payload data. With IPv4 and in IPSec transport mode $1 \mathrm{mb}$ IPSec protected data caused only $0.002 \%, 0.001 \%$, $0.002 \%$ and $0.003 \%$ by AH, ESP-CNF, ESP-ATH, ESPCNF-ATH. The same happened in IPv6 according to the record in table 8 , but it is important to note that for IPv6, since the packet is very large the packet must be fragmented and IPSec header is attached to each fragment. After taking the overall summation the total space overhead will be equal to the (no of fragments * the overhead caused by single fragment) (i.e. as in the case in the record, since it represent the overhead caused by single fragment) this is the reason that explain why IPv6 suffer more IPSec space overhead when large files are involved, since in IPv4 the IPSec header is applied to first fragment only while in IPv6 the header is applied to all.

For the ANOVA of overhead imposed in tunnel mode by the same payload size. The results indicated that protocols and IPsec are significant at $0.1 \%$ level. The means separation using Bonferrion method for the protocols shows IPv4 has the lower overhead as compared to IPv6. While the mean separation of IPsec configuration set, reveals ESP-CNF with the least overhead and the remaining configuration having the same performance matrix

Furthermore, looking down the table, it can be noticed that $100 \mathrm{mb}$ user data caused very little space overhead especially in IPv4 setup because AH, ESP-CNF-ESP-ATH and ESPCNF-ATH add only $0.00002 \%, 0.00001 \%, 0.00001 \%$ and $0.00003 \%$ in transport mode. It is little higher in tunnel mode because in IPSec tunnel mode, the encryption algorithm is applied to the entire IP packet, both the payload and the IP header inclusive, and then encapsulates it in a new IP header, this means that no portion of the IP packet is exempted from IPSec protection during transmission unlike in transport mode where only the IP packet payload is encrypted during transmission.

\section{Conclusion}

This paper demonstrated how IPsec headers under different protocol configurations setup introduced additional processing and space overhead with respect to different file size on two different Internet protocols; IPv4 and IPv6. The study indicated that the cost of IPsec added overhead was smaller when smaller packet sizes were involved for both protocols as compared to larger packet sizes that are IPsec protected with the same configuration as the smaller packet. The only exception was in the cases whereby the packet is very large that it has to be fragmented. In such case IPv6 experienced higher overhead than IPv4. This happened due to the fact that the manner at which IPv6 handles fragmented packet when IPsec is involved was completely different with the way IPv4 tackles its. IPv6 applied IPsec header to all fragmented portion of the packet while IPv4 applied it to the very initial fragment only.

\section{References}

[1] Cheng Min (2011) Research On network Security Based on IPv6 Architecture. 2011 international Conference on Electronics and Optoelectrnics (ICEOE 2011) (PP 1-3) Institute of Electrical Electronics Engineers (IEEE Xplore Digital Library).

[2] Christos Xenakis, Nikolaos Laoutaris, Lazaros Merakos, Ioannis Stavrakakis (2006) A generic characteristics of the overheads imposed by IPSec and associated cryptographic algorithms. ScienceDirect computer networks 50 (2006) 3225-3241

[3] George C. Hadjichristofi Nathaniel J. Davis, IVScott F. Midkiff (2003) IPSec Overhead in Wireline and Wireless Networks for Web and Email Applications.(PP 1-5) IEEE Institute of Electrical Electronics Engineers (IEEEXplore Digital Library).

[4] Eastlake $3^{\text {rd }}$ D. Cryptographic Algorithm Implementation Requirements for Encapsulating Security Payload (ESP) and Authentication Header (AH) IETF RFC 4305 (December 2005)

[5] J. C. Lin, C. T. Chang and W. T. Chung, (2003) "Design, Implementation and Performance Evaluation of IP-VPN", In Proc.of AINA 2003, pp. 206 - 209,

[6] J. Klaue and A. Hess, "On the Impact of IPSec on Interactive Communications" (2005) In Proc. of IPDPS 2005, 8 pp.,

[7] Meenakshi S. P, Raghavan S.V(2006) Impact of IPSec Overhead on Web Application Servers.(PP1-6) Institute of Electrical Electronics Engineers (IEEE Xplore Digital Library).

[8] Mujinga M.H, Muyingi G.S.V.R, Krishna R (2006) IPSec Overhead Analysis in Dual Stack IPv4/IPv6 Transition Mechanisms. (PP 1-6) Institute of Electrical Electronics Engineers (IEEE Xplore Digital Library).

[9] O. Elkeelany, M.M.Matalgah, K.P. Sheikh, G. Chaudhry, D. Medhi and J. Qaddour, (2002)"Performance Analysis of IPSec Protocol: Encryption and Authentication", In Proc. of IEEE Communication Conference ICC2002, Vol. 2, pp. 1164-1168. 
[10] Seiji ARIGA, Masaki MINAMI, Hiroshi ESAKI and Jun MURAI, (2000) "Performance Evaluation of Data Transmission Using IPSec over IPv6 Networks", In Proc. of the 10th Annual Internet Society Conference (INET 2000), Yokohama, Japan.
[11] Todd Lammle (2010) Cisco Certified Network Associate. Wiley Publishing. Inc USA

[12] Wenhong Liu, Zhen Jiang, Hongke Zhang (2006). A Secure Mobile-IPv6 Network Model. ICWMMN 2006 Proceedings (PP 1-4) 